\title{
Multilevel techniques for the solution of HJB minimum-time control problems
}

\author{
Gabriele Ciaramella • Giulia Fabrini
}

Received: date / Accepted: date

\begin{abstract}
The approximation of feedback control via the Dynamic Programming approach is a challenging problem. The computation of the feedback requires the knowledge of the value function, which can be characterized as the unique viscosity solution of a nonlinear Hamilton-Jacobi-Bellman (HJB) equation. The major obstacle is that the numerical methods known in literature strongly suffer when the dimension of the discretized problem becomes large. This is a strong limitation to the application of classical numerical schemes for the solution of the HJB equation in real applications. To tackle this problem, a new multi-level numerical framework is proposed. Numerical evidences show that classical methods have good smoothing properties, which allow one to use them as smoothers in a multilevel strategy. Moreover, a new smoother iterative scheme based on the Anderson acceleration of the classical value function iteration is introduced. The effectiveness of our new framework is proved by several numerical experiments focusing on minimum-time control problems.
\end{abstract}

Keywords Hamilton-Jacobi equation · minimum-time problem · value iteration $\cdot$ policy iteration $\cdot$ Anderson acceleration $\cdot$ multilevel acceleration methods · FAS

Mathematics Subject Classification (2010) 49L20 $\cdot 49 J 15 \cdot 65$ N55

G. Fabrini gratefully acknowledges support by the German Science Fund DFG grant Reduced-Order Methods for Nonlinear Model Predictive Control.

G. Ciaramella

University of Konstanz, Department of Mathematics and Statistics

E-mail: gabriele.ciaramella@uni-konstanz.de

G. Fabrini

University of Konstanz, Department of Mathematics and Statistics

E-mail: giulia.fabrini@uni-konstanz.de 


\section{Introduction}

Many applications in engineering, physics and chemistry deal with minimumtime control problems, whose goal is to compute a control function that is capable to steer a dynamical system from a given initial state to a desired target configuration in the shortest possible time; see, e.g., [19,20,25]. In general, the time needed to reach the target is considered as a cost functional. Hence, a minimum-time problem can be recast in the form of an optimal control problem. In practical applications, it is often desirable to compute minimumtime control functions in a so-called feedback form: the control is a function of the state variable that describes the underlying physical process. A strategy to solve this kind of problems is based on the Dynamic Programming (DP) approach formulated by Bellman [5], which leads to deal with a firstorder non-linear hyperbolic equation, the Hamilton-Jacobi-Bellman equation (HJB). Hence, the construction of the feedback implies the computation of the value function, which can be characterized as the unique viscosity solution of the HJB equation, see [3] and the references therein. The numerical solution of this equation is not an easy task and several methods have been proposed in the literature. The most famous and used ones are the so-called value iteration [11] and policy iteration [18]. The former is based on a simple fixed-point argument and has the main advantage that each iteration requires a low computational effort. The latter is a more sophisticated procedure that has been proved to be equivalent to a generalized Newton method [21]. Each iteration of the policy iteration method requires a higher computational effort if compared to the value iteration, but the convergence rate is much faster; see, e.g., [22].

Based on these two methods, more efficient numerical strategies have been developed in the literature. An interesting idea has been proposed in [1] where the value iteration is used to find a good initialization for the policy iteration. Moreover, different approaches have been proposed in $[8,9]$ and more recently in [12] where the value iteration is used in a domain decomposition type strategy that allows parallel numerical implementations.

The first novelty of this work is the development of a new iteration scheme. This is obtained by a combination of the value iteration procedure and the Anderson acceleration strategy. The latter is an extrapolation method, introduced by Anderson [2], that is capable to accelerate the convergence of fixed-point iteration methods. This technique received a lot of attention in the last years in the field of numerical analysis. In $[29,28]$, the authors prove an equivalence result between the Anderson acceleration strategy and the wellknown preconditioned GMRES; see, e.g., [23]. A general proof of convergence for non-linear differentiable problems is given in [26]. After the development of our accelerated scheme, we extend the existing convergence results [26] to the non-differentiable framework of the Hamilton-Jacobi equation. Numerical evidences show that our strategy is capable to strongly accelerate the value iteration scheme. 
The main drawback of all the methods discussed above (including our new acceleration scheme) is that they strongly suffer when the dimension of the numerical problem becomes large. If an accurate numerical solution is needed, they require a too large computational effort and this makes their use prohibitive. For this reason, we introduce a new multilevel numerical strategy that is capable to solve a given problem of a certain numerical dimension with a much lower computational effort. The development of this new strategy is the main novelty of this work.

Our multilevel approach is based on the idea of the so-called Full Approximation Storage (FAS) scheme; see, e.g., [16,6]. As for classical multigrid methods, a FAS scheme requires at each level a so-called smoother, that is a method capable to reduce the high-frequency error components. In this work, we show by direct numerical evidence that value iteration, policy iteration and Anderson acceleration are efficient smoothers. Therefore, we embed them in a FAS multilevel strategy to obtain new very fast numerical procedures that solve the Hamilton-Jacobi equation within a much lower computational time.

This paper is organized as follows. In Section 2, we briefly recall minimumtime control problems and the Hamilton-Jacobi equation. Section 3 is a short review of the classical value iteration and policy iteration schemes. In Section 4, we present our Anderson acceleration strategy of the value iteration and prove its convergence. Our new multilevel strategy is developed in Section 5. To do so, we first study the smoothing properties of the iterative schemes presented in Sections 3 and 4, and then explain how to use them in combination with a FAS multilevel strategy. The efficiency of our new scheme is shown by several numerical experiments discussed in Section 6. We present our conclusions in Section 7 .

\section{Minimum-time problem and HJ formulation}

Consider the controlled dynamical system

$$
\begin{aligned}
\dot{y}(t) & =f(y(t), u(t)), \quad t>0, \\
y(0) & =x,
\end{aligned}
$$

where $y(t) \in \mathbb{R}^{n}$ is the state variable, $u$ is a control function belonging to $\mathcal{U}:=$ $\left\{u: \mathbb{R}^{+} \rightarrow U\right.$, measurable $\}$ with $U$ a compact subset of $\mathbb{R}^{m}$. If $f: \mathbb{R}^{n} \times U \rightarrow \mathbb{R}^{n}$ is continuous with respect to $(y, u)$ and Lipschitz continuous with respect to the state variable, the Carathéodory theorem $($ see $[3,13])$ guarantees that for any given control function $u \in \mathcal{U}$ and any initial condition $x \in \mathbb{R}^{n}$, there exists a unique trajectory denoted by $y_{x}(t ; u)$ satisfying $(1)$ almost everywhere. Different control policies lead to different trajectories. Hence, one has a family of infinitely many solutions of the controlled system (1) parametrized with respect to $u$. In a minimum-time problem one has to find a control policy leading to a trajectory of (1) such that a given target state $\mathcal{T}$ is reached in the shortest possible time. Let us assume that $\mathcal{T}$ is a compact subset of $\mathbb{R}^{n}$ with 
non-empty interior and piecewise-smooth boundary. The goal of a minimumtime control problem is to find a control function $u \in \mathcal{U}$ that minimizes the quantity

$$
t_{x}(u):= \begin{cases}\inf _{u \in \mathcal{U}}\left\{t \in \mathbb{R}^{+}: y_{x}(t ; u) \in \mathcal{T}\right\} & \text { if } y_{x}(t ; u) \in \mathcal{T} \text { for some } t \\ +\infty & \text { otherwise }\end{cases}
$$

that is the time of arrival to the target starting from the point $x$. Using the quantity $t_{x}(u)$, the so-called value function is defined as

$$
T(x):=\inf _{u \in \mathcal{U}} t_{x}(u)
$$

We observe that the time of arrival to the target can be infinite at some points, as a consequence equation (3) is not defined everywhere if some controllability assumptions are not introduced. This is a free boundary problem where one has to compute at the same time the pair $(T(x), \mathcal{R})$, i.e. the minimumtime function and its domain. However, applying the dynamic-programming principle, and introducing the Kružkov transform [11]

$$
v(x):= \begin{cases}1-\exp (-T(x)) & \text { for } T(x)<+\infty \\ 1 & \text { for } T(x)=+\infty\end{cases}
$$

$v(x)$ can be characterized as the unique viscosity solution of

$$
\begin{aligned}
v(x)+\sup _{u \in U}\{-f(x, u) \cdot \nabla v(x)\} & =1 \text { in } \mathbb{R}^{n} \backslash \mathcal{T}, \\
v(x) & =0 \text { on } \partial \mathcal{T} .
\end{aligned}
$$

We observe that $v(x)$ takes value in $[0,1]$ and this is an important advantage when a numerical solution is required. In many cases, the value function $v$ is defined on $\mathbb{R}^{n}$, but numerically approximated on a subset $\Omega \subset \mathbb{R}^{n}$. Hence, $\Omega$ represents a truncation of the true domain. This truncation can significantly affect the numerical solution and lead to completely wrong results. However, working with (5) and using the boundary condition $v(x)=1$ on $\partial \Omega$ one can control the error of the numerical solution by choosing a sufficiently large domain $\Omega$.

Finally, in our numerical implementations we impose boundary conditions $v(x)=0$ for $x \in \partial \mathcal{T}$ and $v(x)=1$ for $x \in \partial \Omega$; see, e.g., [4,10] for more details on the approximation of minimum-time problems.

\section{Classical iterative solvers for the solution of the Hamilton-Jacobi equation}

Consider a semi-Lagrangian approximation based on a discrete dynamic principle, this leads to the time-discrete scheme

$$
v^{\Delta t}(x)=\min _{u \in U}\left\{e^{-\Delta t} v^{\Delta t}(x+\Delta t f(x, u))+1-e^{-\Delta t}\right\} .
$$


The discrete approximation $v^{\Delta t}(x)$ converges to the solution $v(x)$ as $\Delta t \rightarrow 0$; see, e.g., [11] and the references therein. Notice that in (6) the term $e^{-\Delta t}$ is sometimes replaced by its first-order Taylor approximation $1-\Delta t$. Projecting the time discrete equation (6) on a space grid $\mathcal{G}$ whose elements are denoted by $x_{i}$, we obtain a fully discrete equation

$$
v_{i}^{\Delta t}=\min _{u \in U}\left\{e^{-\Delta t} \mathcal{I}\left[v_{i}^{\Delta t}\right]\left(x_{i}+\Delta t f\left(x_{i}, u\right)\right)+1-e^{-\Delta t}\right\}, \quad \text { for } i=1,2, \ldots
$$

Without loss of generality, we assume for simplicity that $\mathcal{G}$ is a uniform space grid with step size $\Delta x$. Extensions to nonuniform grids can be performed in a straightforward manner. Note that in general, the point $x_{i}+\Delta t f\left(x_{i}, u\right)$ is not a node of the grid $\mathcal{G}$, and the value $v^{\Delta t}\left(x_{i}+\Delta t f\left(x_{i}, u\right)\right)$ is approximated by $\mathcal{I}\left[v_{i}^{\Delta t}\right]\left(x_{i}+\Delta t f\left(x_{i}, u\right)\right)$, where $\mathcal{I}\left[v_{i}^{\Delta t}\right]$ is an interpolation operator. Let $V$ be an array whose entries are real values associated to the points $x_{i} \in \mathcal{G}$, that is $V:=\left\{v_{i}^{\Delta t} \in \mathbb{R}:\right.$ for all $i$ such that $\left.x_{i} \in \mathcal{G}\right\}$. Notice that $V$ is a vector in $1 \mathrm{D}$, a matrix in 2D and a tensor in ND. Moreover, if one denotes by $N$ the cardinality of $\mathcal{G}$, then $V$ can be regarded as a vector in $\mathbb{R}^{N}$. If we introduce the operator $S: V \mapsto S(V)$ whose $i^{\text {th }}$ entry is

$$
(S(V))_{i}:=\min _{u \in U}\left\{e^{-\Delta t} \mathcal{I}\left[v_{i}^{\Delta t}\right]\left(x_{i}+\Delta t f\left(x_{i}, u\right)\right)+1-e^{-\Delta t}\right\},
$$

then equation (7) can be written as the fixed-point problem

$$
V=S(V) \text {. }
$$

The accuracy of the discretization scheme (9)-(8) is extensively discussed in the literature; see, e.g., [11, Chapters 8 and 9$]$ and references therein. In particular, it is possible to prove that there exists a constant $C>0$ independent of $\Delta t$ and $\Delta x$ such that

$$
\left|v_{i}^{\Delta t}-v\left(x_{i}\right)\right| \leq C \sqrt{\Delta t}\left(1+\left(\frac{\Delta x}{\Delta t}\right)^{2}\right),
$$

where $v\left(x_{i}\right)$ is the continuous solution to (5) restricted to the points $x_{i} \in \mathcal{G}$.

In the last decades, several numerical methods have been developed for the solution to the nonlinear fixed-point problem (9). The most used and general ones, the so called value iteration and policy iteration, are discussed in the next paragraphs.

3.1 Value iteration

A natural way to solve (7) is to use a fixed-point iteration

$$
V^{k+1}=S\left(V^{k}\right), \text { for } k=0,1,2, \ldots
$$

This is the well-known value iteration (VI) scheme. The corresponding numerical implementation is given in Algorithm 1. It can be shown that $S$ is a 


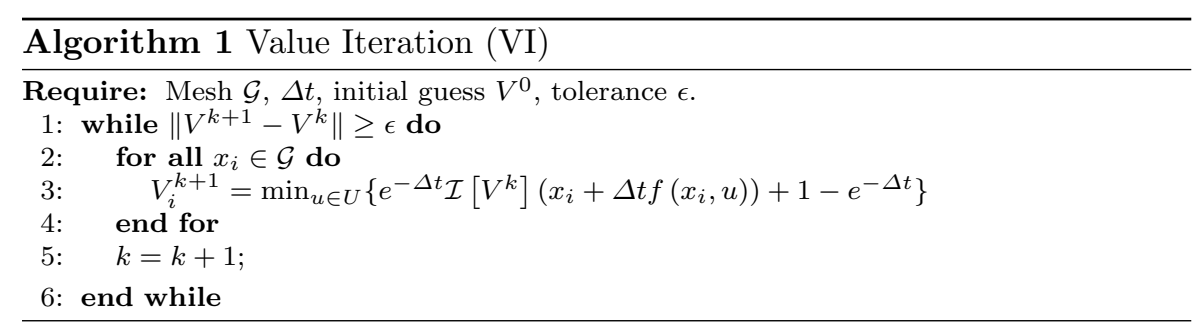

contraction for any initial guess $V^{0}$ with a contraction coefficient $e^{-\Delta t}$. Thus, for any arrays $V$ and $W$ defined on the grid $\mathcal{G}$ it holds that

$$
\|S(V)-S(W)\| \leq e^{-\Delta t}\|V-W\|,
$$

where $\|V\|:=\max _{x_{i} \in \mathcal{G}}\left|V_{i}\right|$ is the usual vector $\infty$-norm. Note that the same norm is used in Algorithm (1), step 1.

\subsection{Policy iteration}

We now consider a very popular method for solving (9), the well-known policy iteration (PI) procedure. First, an initial guess $V^{0}$ for the value function is chosen. This allows us to compute a new control policy. Once the new control has been obtained one has to solve a linear (if the interpolation operator is linear) system to obtain the new value function $V^{1}$. This step is repeated until convergence. The PI procedure for the minimum-time problem is given in Algorithm 2. Since we assume that the interpolation operator $\mathcal{I}$ is linear, in

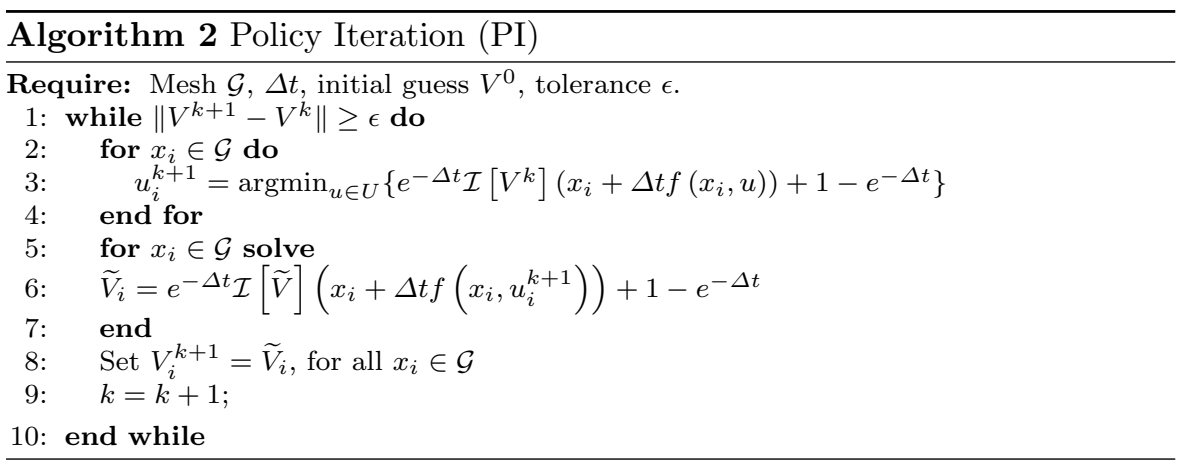

step 3 we have to solve a linear system. This is done with a stationary fixedpoint method. The PI method corresponds to the Newton method applied to the equation (9) of dynamic programming. The interested reader can find in [22] the convergence analysis; see also [24,21]. 


\section{Anderson Acceleration}

The Anderson acceleration method is an extrapolation technique used to accelerate a fixed-point iteration method. This strategy was introduced in 1965 by Anderson [2]. We use Anderson's technique to accelerate the VI (Algorithm $1)$. Let $w \in \mathbb{R}^{N}$ be a representation of an array defined on the grid $\mathcal{G}$, we reformulate $(9)$ as $w=S(w)$. Denote by $w_{j} \in \mathbb{R}^{N}$ the approximations to a solution $w$ obtained by the Anderson method at iterations $j=1, \ldots, k$, and by $g_{j}:=G\left(w_{j}\right):=S\left(w_{j}\right)-w_{j}$ the corresponding residuals. Let $m \in \mathbb{N}^{+}$and define $m_{k}:=\min (m, k)$. According to the Anderson acceleration strategy, the new approximation $w_{k+1}$ is computed as

$$
w_{k+1}=\left(1-\beta_{k}\right) \sum_{j=0}^{m_{k}} \alpha_{j}^{k} w_{k-m_{k}+j}+\beta_{k} \sum_{j=0}^{m_{k}} \alpha_{j}^{k} S\left(w_{k-m_{k}+j}\right),
$$

where the coefficients $\alpha_{j}^{k}$ for $j=0, \ldots, m_{k}$ are obtained as the solution to the following minimization problem

$$
\min _{\alpha_{0}^{k}, \ldots, \alpha_{m_{k}}^{k}}\left\|\sum_{j=0}^{m_{k}} \alpha_{j}^{k} g_{k-m_{k}+j}\right\| \quad \text { s.t. } \sum_{j=0}^{m_{k}} \alpha_{j}^{k}=1,
$$

for some norm $\||\cdot|\|$. The parameters $\beta_{j}>0$ for $j=0, \ldots, k$ are relaxation parameters that are used to improve the convergence behavior of the Anderson acceleration method $[29,28]$. However, their choice is in general heuristic and strongly depending on the problem. Therefore, we consider here only $\beta_{k}=1$; see also $[29,28]$.

Algorithm 3 shows a possible implementation corresponding to the Anderson acceleration. Notice that iterations require the storage of the history of

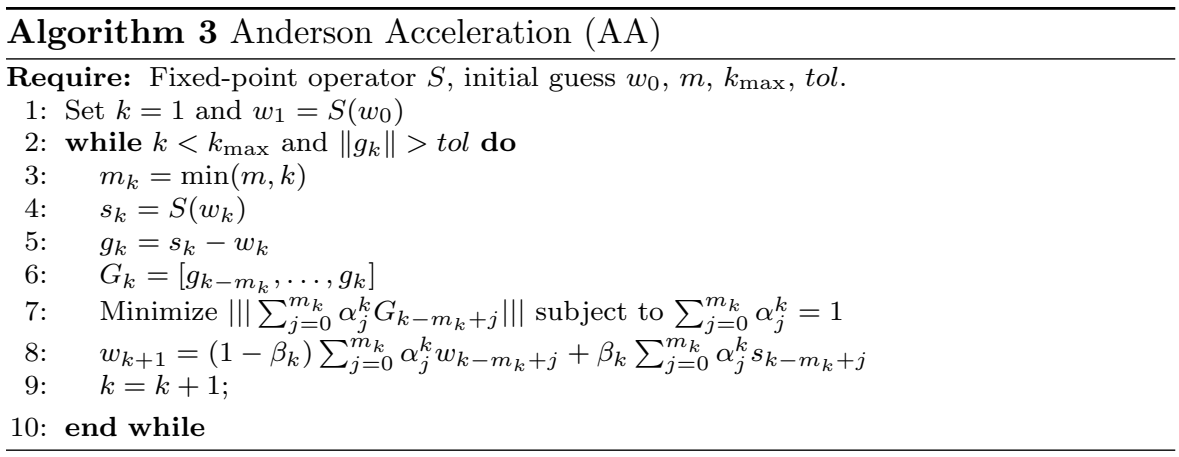

residuals of depth at most $m_{k}+1$. This fact is crucial when the dimension $N$ is large. In this case one has to consider small values of $m$ to reduce the amount of information stored during the iterations.

A crucial step in the Anderson strategy is the solution of the minimization problem (13), where any norm could be used. Typically one uses the Euclidean 
norm, so the first-order sufficient optimality condition for the minimization problem is a linear system. Different approaches are considered to solve (13); see, e.g., [14]. An efficient implementation of Anderson algorithm is proposed in [28], where (13) is transformed into an unconstrained problem. To do so, one defines $\Delta g_{i}=g_{i+1}-g_{i}$ for each $i$ and set $\widetilde{G}_{k}=\left[\Delta g_{k-m_{k}}, \ldots, \Delta g_{k-1}\right]$. Then the least squares problem (13) is equivalent to

$$
\min _{\gamma=\left[\gamma_{0}, \ldots, \gamma_{m_{k}-1}\right]^{\top}}\left\|g_{k}-\widetilde{G}_{k} \gamma\right\|_{2} \text {. }
$$

Notice that the coefficients $\alpha_{j}$ in (13) and $\gamma_{j}$ are related by $a_{0}=\gamma_{0}, \alpha_{i}=$ $\gamma_{i}-\gamma_{i-1}$ for $1 \leq i \leq m_{k}-1$ and $\alpha_{m_{k}}=1-\gamma_{m_{k}-1}$. Moreover, if $m_{k}$ is too large, the matrix $\widetilde{G}_{k}$ could be ill-conditioned. For this reason we consider a sufficiently small $m_{k}$. The unconstrained least-squares problem (14) leads to a modified form of Anderson acceleration given in Algorithm (4), where the least-squares solution is denoted by $\gamma^{k}=\left[\gamma_{0}^{k}, \ldots, \gamma_{m_{k}-1}^{k}\right]^{\top} \in \mathbb{R}^{m_{k}}$, and we have

$$
w_{k+1}=S\left(w_{k}\right)-\sum_{i=0}^{m_{k}-1} \gamma_{i}^{k}\left[S\left(w_{k-m_{k}+i-1}\right)-S\left(w_{k-m_{k}+i}\right)\right]=S\left(w_{k}\right)-\mathcal{S}_{k} \gamma^{k},
$$

where $\mathcal{S}_{k}=\left[\Delta S_{k-m_{k}}, \ldots, \Delta S_{k-1}\right] \in \mathbb{R}^{m_{k} \times m_{k}}$ with $\Delta S_{i}=S\left(w_{i+1}\right)-S\left(w_{i}\right)$ for each $i$.

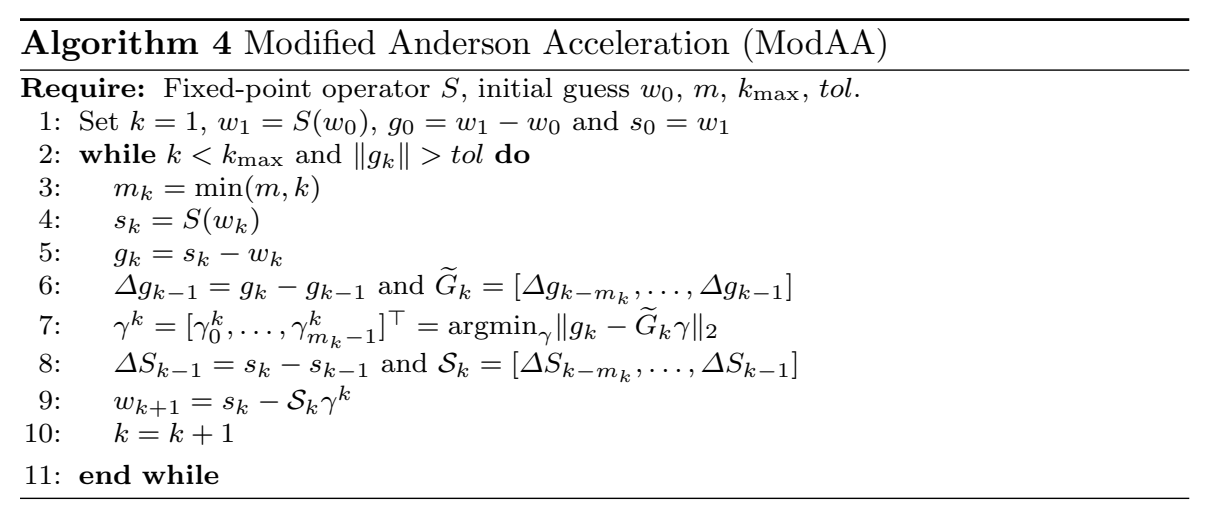

As the algorithm proceeds, the successive least-squares problems in step 7 are solved by a QR-factorization. In particular, one first decomposes $\widetilde{G}_{k}=$ $Q_{k} R_{k}$ with a factorization ("economy-size"). Then the solution $\gamma^{k}$ is obtained as $\gamma^{k}=R_{k}^{-1}\left(Q_{k}^{\top} g_{k}\right)$. In this step, the QR-factorization can be solved efficiently. In fact, suppose that the factorization $\widetilde{G}_{k}=Q_{k} R_{k}$ is known and that one has to compute the decomposition $\widetilde{G}_{k+1}=Q_{k+1} R_{k+1}$. Since $\widetilde{G}_{k+1}$ is obtained by adding one column to $\widetilde{G}_{k}$, that is $\widetilde{G}_{k+1}=\left[\widetilde{G}_{k}, \Delta g_{k}\right]$, then the factors $Q_{k+1}$ and $R_{k+1}$ can be obtained by adding one column to $Q_{k}$ and $R_{k}$, respectively. This can be achieved by a single modified Gram-Schmidt sweep. This procedure is detailed in Algorithm 5; see also [28]. 


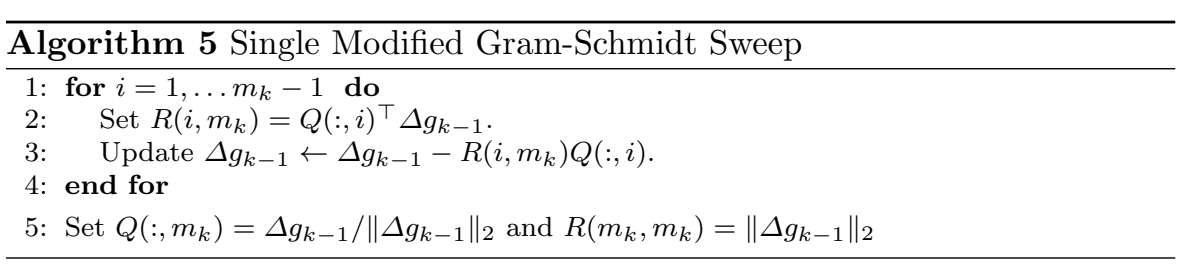

Convergence results

In this section, we prove local convergence result of the Anderson acceleration procedure in the case where the fixed-point operator $S$ is given by (8). To do so, we recall that there exists $v^{*} \in \mathbb{R}^{N}$ such that $G\left(v^{*}\right)=S\left(v^{*}\right)-v^{*}=0$ and assume that there is $M_{\alpha}$ such that for all $k \geq 0$ it holds

$$
\sum_{j=1}^{m_{k}}\left|\alpha_{j}\right| \leq M_{\alpha}
$$

Moreover, recalling (12) and that $\|\cdot\|$ denotes the usual $\infty$-norm, we have that there is $c \in(0,1)$ such that for all $w, v \in \mathcal{B}(\hat{\rho}):=\left\{v \mid\left\|v-v^{*}\right\| \leq \hat{\rho}\right\}$ for some $\hat{\rho}>0,\|S(w)-S(v)\| \leq c\|w-v\|$, with $c=e^{-\Delta t}$. Finally, we make the following assumption

$$
\left\|\sum_{j=0}^{m_{k}} \alpha_{j}^{k} G\left(w_{k-m_{k}+j}\right)\right\| \leq\left\|G\left(w_{k}\right)\right\|
$$

A general local convergence analysis is given in [26], where the fixed-point operator is assumed to be Lipschitz continuously differentiable. However, the operator $S$ defined in (8) is not differentiable because of the min function. Therefore, we need to extend the result given in [26]. To do so, we first recall some definitions and technical results about semismooth functions.

Definition 1 Let a function $f: W \rightarrow \mathbb{R}^{m}$ be defined on an open set $W \subset \mathbb{R}^{N}$. Then, for $0<\alpha \leq 1, f$ is called $\alpha$-order semismooth at $x \in W$ if $\mathrm{f}$ is locally Lipschitz continuous near $x$, the directional derivative $f^{\prime}(x, \cdot)$ exists, and

$$
\sup _{M \in \partial f(x+s)}\left\|M s-f^{\prime}(x, s)\right\|=O\left(\|s\|^{1+\alpha}\right) \quad \text { as } s \rightarrow 0,
$$

where $\partial f(x+s)$ is the subdifferential of $f$ at $x+s$. If $f$ is $\alpha$-order semismooth at all $x \in W$, we call $f \alpha$-order semismooth (on $W$ ).

For $\alpha$-order semismooth functions, the following results hold; see [27, Chapter $2]$.

Proposition 1 Let $f: W \rightarrow \mathbb{R}^{m}$ be defined on the open set $W \subset \mathbb{R}^{N}$. Then for $x \in W$ and $0<\alpha \leq 1$ the following statements are equivalent:

(a) $f$ is $\alpha$-order semismooth at $x$. 
(b) $f$ is Lipschitz continuous near $x, \alpha$-order B-differentiable ( "B" stands for Bouligand, see Definition 2.1 in [27]) at $x$, and

$$
\sup _{M \in \partial f(x+s)}\|f(x+s)-f(x)-M s\|=O\left(\|s\|^{1+\alpha}\right) \text { as } s \rightarrow 0 .
$$

Definition 2 A function $f: W \rightarrow \mathbb{R}^{m}$ defined on the open set $W \subset \mathbb{R}^{N}$ is called $P C^{k}$-function, $1 \leq k \leq \infty$, if $f$ is continuous and if at every point $x \in W$ there exist a neighborhood $X \subset W$ of $x$ and a finite collection of $C^{k}$-functions $f^{i}: X \rightarrow \mathbb{R}^{m}, i=1, \ldots, M$, such that

$$
f(x) \in\left\{f^{1}(x), \ldots, f^{M}(x)\right\} \quad \forall x \in X .
$$

Proposition 2 Let $f: W \rightarrow \mathbb{R}^{m}$ be a $P C^{2}$-function on the open set $W \subset$ $\mathbb{R}^{N}$. Then $f$ is 1 -order semismooth.

Lemma 1 Let $U$ be a discrete set of admissible controls: $U:=\left\{u_{1}, \ldots, u_{m}\right\}$ with $u_{j} \in \mathbb{R}$ for $j=1, \ldots, m$. Then $S$ is 1-order semismooth.

Proof Let $\widetilde{V}(u, v)$ be defined as

$$
(\widetilde{V}(u, v))_{j}:=e^{-\Delta t} I[v]\left(x_{j}+\Delta t f\left(x_{j}, u\right)\right)+\left(1-e^{-\Delta t}\right)
$$

for $j=1, \ldots, N$. Since the operator $S: \mathbb{R}^{n} \rightarrow \mathbb{R}^{n}$ is given by $v \mapsto(S(v))_{j}:=$ $\min _{u \in U}(\widetilde{V}(u, v))_{j}$ and $U$ is a set of $m<\infty$ elements, we have

$$
S(v)=\min \left\{\widetilde{V}\left(u_{1}, v\right), \ldots, \widetilde{V}\left(u_{m}, v\right)\right\} .
$$

Notice that the map $v \mapsto \widetilde{V}\left(u_{j}, v\right)$ for $j=1, \ldots, m$ is $C^{\infty}$ for any $u_{j}$. Therefore, $v \mapsto S(v)$ is $P C^{2}$ according to the Definition 2, and Proposition 2 implies that it is 1 -order semismooth.

Consider the map $G: \mathbb{R}^{N} \rightarrow \mathbb{R}^{N}$ defined by $G(v):=S(v)+v$. Let $\partial S\left(v^{*}\right) \subset$ $\mathbb{R}^{N \times N}$ denote the subdifferential of $S$ at $v^{*}$ and $\partial G\left(v^{*}\right) \subset \mathbb{R}^{N \times N}$ the subdifferential of $G$ at $v^{*}$. Consider any element $S^{*} \in \partial S\left(v^{*}\right)$ and $G^{*}=S^{*}+I \in \partial G\left(v^{*}\right)$, where $I$ is the identity matrix. Notice that the Lipschitz continuity of $S$ implies that $\left\|S^{\prime}(v)\right\| \leq c<1$, for any $S^{\prime} \in \partial S(v)$, for all $v \in \mathcal{B}(\hat{\rho})$. Moreover, we define the error $e:=v-v^{*}$.

Lemma 2 For any $v \in \mathbb{R}^{N}$ it holds that

$$
\begin{aligned}
\left\|G(v)-G^{*} e\right\| & =O\left(\|e\|^{2}\right), \\
\|e\|(1-c) \leq\|G(v)\| & \leq(1+c)\|e\| .
\end{aligned}
$$

Proof The statement (17) follows from Lemma 1 which guarantees that $S$ is 1-order semismooth and the statement (b) of Proposition 1. Next, we prove the right inequality of (18). Since $G\left(v^{*}\right)=0$, we have

$\|G(v)\|=\left\|G(v)-G\left(v^{*}\right)\right\|=\left\|S(v)-v-S\left(v^{*}\right)+v^{*}\right\| \leq\left\|S(v)-S\left(v^{*}\right)\right\|+\left\|v-v^{*}\right\|$, and the result follows by recalling that $S$ is Lipschitz with Lipschitz constant $c$. The lower bound of $\|G(v)\|$ follows by the reverse triangle inequality. 
Notice that (17) implies that there exist a $\rho>0$ and a $\gamma>0$ such that

$$
\left\|G(v)-G^{*} e\right\| \leq \frac{\gamma}{2}\|e\|^{2}
$$

for all $v$ such that $e=v-v^{*} \in \mathcal{B}(\rho)$.

We are now ready to extend the convergence result given in [26].

Theorem 1 Let (15) holds and let $c<\widehat{c}<1$. If the initial guess $v_{0} \in \mathbb{R}^{N}$ is sufficiently close to $v^{*}$, then the sequence $\left(v_{k}\right)_{k \in \mathbb{N}}$ generated by the Anderson iterations converges to $v^{*}$ in the sense that

$$
\begin{aligned}
\left\|G\left(v_{k}\right)\right\| & \leq \widehat{c}^{k}\left\|G\left(v_{0}\right)\right\|, \\
\left\|v_{k}-v^{*}\right\| & \leq \frac{1+c}{1-c} \widehat{c}^{k}\left\|v_{0}-v^{*}\right\| .
\end{aligned}
$$

Proof Let $v_{0} \in \mathcal{B}(\rho)$ for some $\rho$. Consider the sequence $\left(v_{k}\right)_{k \in \mathbb{N}}$ and define the error sequence $\left(e_{k}\right)_{k \in \mathbb{N}}$ by $e_{k}:=v_{k}-v^{*}$. The inequality (21) follows from $(20)$ and Lemma 2 . Hence, we have to prove (20). To do so, consider $\rho$ sufficiently small so that $\rho<2(1-c) / \gamma$ and

$$
\frac{\left(\frac{c}{\hat{c}}+\frac{M_{\alpha} \gamma \rho}{2(1-c)} \widehat{c}^{-m-1}\right)}{1-\frac{\gamma \rho}{2(1-c)}} \leq 1
$$

where $\gamma$ is as in (19), $M_{\alpha}$ is given in (15) and $c$ is the Lipschitz constant of $S$. Since $v_{0}$ is sufficiently close to $v^{*}$, we can consider that $\left\|e_{0}\right\|$ is sufficiently small and such that

$$
\left(\frac{M_{\alpha}(c+\gamma \rho / 2)}{1-c}\right) \widehat{c}^{-m}\left\|G\left(v_{0}\right)\right\| \leq\left(\frac{M_{\alpha}(1+c)(c+\gamma \rho / 2)}{1-c}\right) \widehat{c}^{-m}\left\|e_{0}\right\| \leq \rho .
$$

We will proceed by induction. Assume that (20) holds for $k=0, \ldots K$. Since $v_{0}$ is chosen sufficiently close to $v^{*}$, we can consider that $\left\|e_{0}\right\| \leq \frac{1-c}{1+c} \rho$. The induction hypothesis (20) implies that (21) holds for $k=0, \ldots, K$. Therefore, using also that $\widehat{c}<1$ we have $\left\|e_{k}\right\| \leq \frac{1+c}{1-c} \widehat{c}^{k}\left\|e_{0}\right\| \leq \widehat{c}^{k} \rho \leq \rho$, for $k=1, \ldots, K$. Hence, (17) implies that $G\left(v_{k}\right)=G^{*} e_{k}+\Delta_{k}$, where

$$
\left\|\Delta_{k}\right\| \leq \frac{\gamma}{2}\left\|e_{k}\right\|^{2}
$$

This implies that

$$
S\left(v_{k}\right)=v^{*}+S^{*} e_{k}+\Delta_{k} .
$$

Define $\bar{\Delta}_{K}:=\sum_{j=0}^{m_{K}} \alpha_{j}^{K} \Delta_{K-m_{K}+j}$. Using (25) and that $\sum_{j=0}^{m_{K}} \alpha_{j}^{K}=1$, we have

$$
\begin{aligned}
v_{K+1} & =\sum_{j=0}^{m_{K}} \alpha_{j}^{K} S\left(v_{K-m_{K}+j}\right)=\sum_{j=0}^{m_{K}} \alpha_{j}^{K}\left(v^{*}+S^{*} e_{K-m_{K}+j}+\Delta_{K-m_{K}+j}\right) \\
& =v^{*}+\sum_{j=0}^{m_{K}}\left(\alpha_{j}^{K} S^{*} e_{K-m_{K}+j}\right)+\bar{\Delta}_{K} .
\end{aligned}
$$


Next, we estimate $\bar{\Delta}_{K}$. To do so, we first notice that (24) and (26) imply that

$$
\left\|\bar{\Delta}_{K}\right\| \leq \sum_{j=0}^{m_{K}}\left|\alpha_{j}^{K}\right| \gamma\left\|e_{K-m_{K}+j}\right\|^{2} / 2 .
$$

Lemma 2 , the induction hypothesis (20) and $\left\|e_{k}\right\| \leq \rho$ for $k=0, \ldots, K$, and the fact that $K-m_{K}+j=K-\min (m, K)+j \geq K-m$, imply

$$
\begin{aligned}
\left\|e_{K-m_{K}+j}\right\|^{2} & \leq\left\|e_{K-m_{K}+j}\right\| \frac{1}{1-c}\left\|G\left(v_{K-m_{K}+j}\right)\right\| \leq \frac{\rho}{1-c}\left\|G\left(v_{K-m_{K}+j}\right)\right\| \\
& \leq \frac{\rho}{1-c} \widehat{c}^{K-m_{K}+j}\left\|G\left(v_{0}\right)\right\| \leq \frac{\rho}{1-c} \widehat{c}^{K-m}\left\|G\left(v_{0}\right)\right\| .
\end{aligned}
$$

Hence, using (27) and (15), we obtain

$$
\begin{aligned}
\left\|\bar{\Delta}_{K}\right\| & \leq \sum_{j=0}^{m_{K}}\left|\alpha_{j}^{K}\right| \gamma \frac{\left\|e_{K-m_{K}+j}\right\|^{2}}{2} \leq \sum_{j=0}^{m_{K}}\left|\alpha_{j}^{K}\right| \frac{\gamma}{2} \frac{\rho}{1-c} \widehat{c}^{K-m}\left\|G\left(v_{0}\right)\right\| \\
& \leq \frac{M_{\alpha} \gamma \rho}{2(1-c)} \widehat{c}^{K-m}\left\|G\left(v_{0}\right)\right\| \leq \frac{M_{\alpha} \gamma \rho}{2(1-c)} \widehat{c}^{-m}\left\|G\left(v_{0}\right)\right\| .
\end{aligned}
$$

The induction hypothesis implies that for $0 \leq j \leq m_{k}$ one has

$$
\left\|e_{K-m_{K}+j}\right\| \leq \frac{1}{1-c}\left\|G\left(v_{K-m_{K}+j}\right)\right\| \leq \frac{\widehat{c}^{K-m_{K}+j}}{1-c}\left\|G\left(v_{0}\right)\right\| \leq \frac{\widehat{c}^{-m}}{1-c}\left\|G\left(v_{0}\right)\right\| .
$$

Hence, by (29), and recalling that $\sum_{j=0}^{m_{K}} \alpha_{j}^{K}=1$ and $\left\|S^{*}\right\| \leq c \leq 1$, we get

$$
\left\|\sum_{j=0}^{K} \alpha_{j}^{K} S^{*} e_{K-m_{K}+j}\right\| \leq \sum_{j=0}^{K}\left|\alpha_{j}\right|\left\|S^{*}\right\|\left\|e_{K-m_{K}+j}\right\| \leq \frac{M_{\alpha} c}{1-c} \widehat{c}^{-m}\left\|G\left(v_{0}\right)\right\| .
$$

Now, we rewrite (26) as $e_{K+1}=\sum_{j=0}^{m_{K}}\left(\alpha_{j}^{K} S^{*} e_{K-m_{K}+j}\right)+\bar{\Delta}_{K}$, and combining (26), (28) and (30) we obtain

$$
\left\|e_{K+1}\right\| \leq\left\|G\left(v_{0}\right)\right\|\left(\frac{M_{\alpha}(c+\gamma \rho / 2)}{1-c}\right) \widehat{c}^{-m} \leq \rho,
$$

where the last inequality follows from (23). Since $\left\|e_{K+1}\right\| \leq \rho \leq \hat{\rho}$, we may apply (25) with $k=K+1$ to obtain $G\left(v_{K+1}\right)=\left(S^{*}-I\right) e_{K+1}+\Delta_{K+1}$ with

$$
\left\|\Delta_{K+1}\right\| \leq \frac{\gamma}{2}\left\|e_{K+1}\right\|^{2},
$$

which follows by Lemma 2 and (19). Since $S^{*}$ and $S^{*}-I$ commute we have

$$
\begin{aligned}
G\left(v_{K+1}\right) & =\left(S^{*}-I\right) e_{K+1}+\Delta_{K+1} \\
& =\left(S^{*}-I\right)\left[\sum_{j=0}^{m_{K}}\left(\alpha_{j}^{K} S^{*} e_{K-m_{K}+j}\right)+\bar{\Delta}_{K}\right]+\Delta_{K+1} \\
& =S^{*} \sum_{j=0}^{m_{K}} \alpha_{j}^{K} G\left(v_{K-m_{K}+j}\right)-\bar{\Delta}_{K}+\Delta_{K+1} .
\end{aligned}
$$


Using (31), (32) and (21), we obtain $\left\|\Delta_{K+1}\right\| \leq \frac{\gamma \rho}{2(1-c)}\left\|G\left(v_{K+1}\right)\right\|$. This estimate, the induction hypothesis $(20),(16), 3,(28)$, and (33) imply that

$$
\begin{aligned}
\left\|G\left(v_{K+1}\right)\right\|\left(1-\frac{\gamma \rho}{2(1-c)}\right) & \leq\left\|G\left(v_{K+1}\right)\right\|-\left\|\Delta_{K+1}\right\| \\
& \leq c\left\|\sum_{j=0}^{m_{K}} \alpha_{j}^{K} G\left(v_{K-m_{K}+j}\right)\right\|+\left\|\bar{\Delta}_{K}\right\| \\
& \leq\left(\frac{c}{\widehat{c}}+\left(\frac{M_{\alpha} \gamma \rho}{2(1-c)}\right) \widehat{c}^{-m-1}\right) \widehat{c}^{K+1}\left\|G\left(v_{0}\right)\right\|,
\end{aligned}
$$

and the claim follows recalling (22).

\section{Multilevel acceleration methods}

The methods presented in Sections 3 and 4 perform very well if used to solve discrete problems defined on coarse grids, but their computational costs become too large when one needs an accurate solution on a very fine grid. This issue makes the use of these iterative methods prohibitive. However, as we are going to see in this section, these methods possess a very interesting property: they are very effective when dealing with high-frequencies error components, that are highly oscillating components of the error. This property is discussed in Section 5.1 and means that VI, PI and AA can be considered as "smoothers". Therefore, they can be successfully used in a multilevel setting, as described in Section 5.2 .

\subsection{Smoothing property of VI, PI and AA}

In this section, we present a qualitative analysis to describe the "smoothing" property of VI, PI and AA. To do so, let us consider a two-dimensional minimum-time control problem, where the dynamics is given by

$$
f(x, y, u)=\left[\begin{array}{l}
\cos (u) \\
\sin (u)
\end{array}\right] \text {. }
$$

The computational domain $\Omega$ is approximated by a uniform grid of $n_{x}^{2}$ nodes denoted by $\left(x_{i}, y_{j}\right), i, j=1, \ldots, n_{x}$ with step size $\Delta x$.

The key idea of our qualitative analysis is to expand the discrete numerical error $e^{k}$ in discrete-sine Fourier series:

$$
e^{k}=\sum_{i, j=1}^{n_{x}} c_{i, j}^{k} \varphi_{i, j}, \quad c_{i, j}^{k}=\left\langle e^{k}, \varphi_{i, j}\right\rangle, i, j=1, \ldots, n_{x},
$$

where $\langle\cdot, \cdot\rangle$ is the Euclidean scalar product and the orthonormal basis vectors $\varphi_{i, j}$ are given by

$$
\varphi_{i, j}=\sqrt{\frac{2}{n_{x}+1}} \sin \left(\frac{i \ell \pi}{n_{x}+1}\right) \sin \left(\frac{j m \pi}{n_{x}+1}\right) \quad \ell, m=1, \ldots, n_{x} .
$$



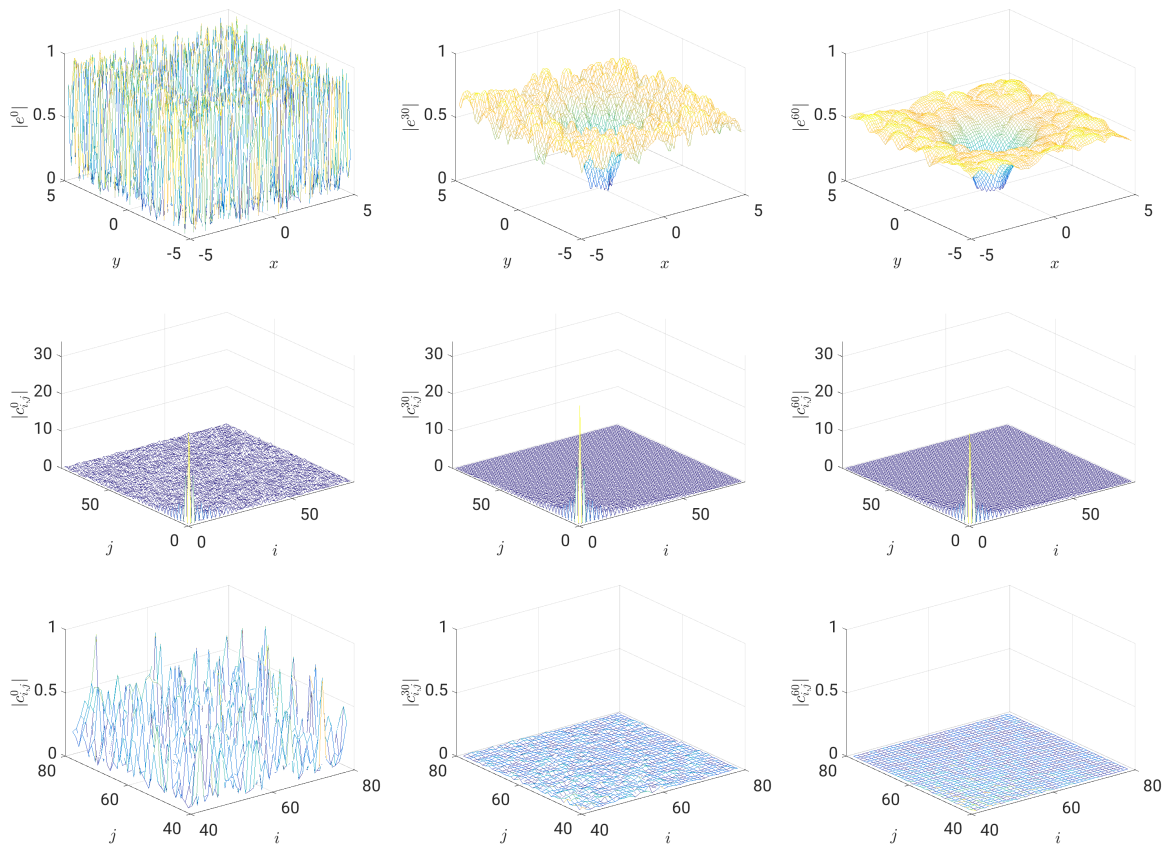

Fig. 1 First row: error at iteration $k=0$ (left), $k=30$ (middle), $k=60$ (right), second row: coefficients $c_{i, j}$ at iteration $k=0$ (left), $k=30$ (middle), $k=60$ (right), coefficients before the high-frequency components, at iteration $k=0$ (left), $k=30$ (middle), $k=60$ (right).

We then study the decay of the coefficients $c_{i, j}^{k}$ as the iterations proceed. To do so, we choose a random initial condition, perform few iterations of VI, PI and AA, and analyze the decay of the Fourier coefficients.

The effect of the smoothing is clearly shown in Figure 1 (first row), where the approximations obtained by the VI at iterations $k=0, k=30$ and $k=60$ are depicted. We observe that at iteration $k=0$ the error is everywhere highly oscillating (Figure 1, top left), while after few iterations the error is much smoother and hence dominated by low-frequency components (Figure 1, top middle and top right). This is very clearly shown in Figure 1 (middle row and bottom row), where the absolute value of the coefficients $c_{i, j}$ is depicted. It is evident that the dominating coefficients are the ones corresponding to the low frequencies ( $c_{i, j}$ for "small" $i$ and $j$ ). Moreover, we observe that the magnitude of the low-frequency coefficients decays very slowly (middle row) as a function of the iteration count $k$, while the high-frequency coefficients are very quickly reduced to very small values (bottom row). The same behavior can be observed by performing the same experiment using PI or AA.

Next, we compare the smoothing process of the three methods VI, PI and AA. We focus our attention on the behavior of the high-frequency coefficients at iteration $k=1$ and $k=4$ and show them in Figure 2. We observe that after one iteration of PI the coefficients are already close to zero (Figure 2, top 

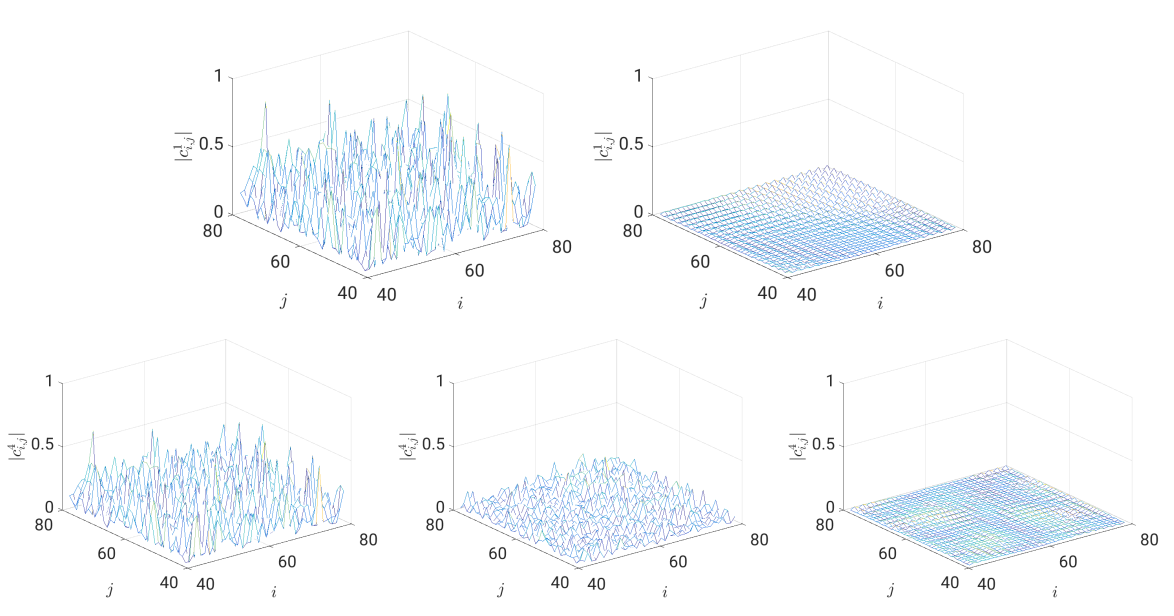

Fig. 2 Coefficients $c_{i, j}$ before the high-frequency components after a single iteration: VI (first row, left), PI (first row, right) and after 4 iterations: VI (second row, left), AA (second row, middle), PI (second row, right).

right), while after a single iteration of VI the coefficients oscillate between 0 and 0.8 (Figure 2, top left). We remark that after a single iteration, AA and VI leads to the same approximation. Hence, the coefficients obtained using AA as smoother coincide with the ones obtained using VI. In Figure 2 bottom row, we plot the same Fourier coefficients after four iterations of the three methods. We clearly observe that the PI is the best smoother, since the corresponding highfrequency coefficients are already very close to zero after only four iterations (Figure 2, bottom right). The slowest method is the VI, which is capable to reduce the Fourier coefficients to about 0.6 (Figure 2, bottom left). The AA method shows an intermediate behavior and is capable to reduce the Fourier coefficients to about 0.2 (Figure 2 , bottom middle). In conclusion, AA is a more efficient smoother than VI, while PI is the best smoother, and all the three methods can be used in a multilevel setting.

\subsection{Full Approximation Storage Scheme}

The idea of a multilevel approach is to compute the solution of a problem by iteratively correcting high-frequency and low-frequency components of the error on different levels (or grids). The high-frequency error components are corrected on finer grids by computationally cheap iterative procedures called smoothers. As we have seen in Section 5.1, few iterations of a smoother are in general sufficient to reduce the high-frequency error components and leave only smooth (low-frequency) error components. The low-frequency components can be accurately represented as the solution of a coarser system that can be solved by exploiting accurate methods, whose use is computationally possible since they are applied to very coarse problems. Once this coarser problem is solved, 
its solution is interpolated back to the fine grid to correct the fine-grid approximation for its low-frequency errors. Applying this method recursively to solve the coarse-grid problem leads to a multilevel strategy. When the underlying problem is non-linear, one refers to this multilevel idea as "full approximation storage" (FAS) scheme; see, e.g., [16,6,17].

In Section 5.1, we have seen that the VI, PI and AA methods have very good smoothing properties. Therefore, it is natural to embed them in a multilevel procedure and obtain very efficient multigrid FAS methods for the efficient solution of the non-linear Hamilton-Jacobi equation (9).

To do so, let us introduce a sequence of grids $\Omega_{\ell}$ with step size $\Delta x_{\ell}$, so that $\Delta x_{\ell-1}=2 \Delta x_{\ell}$ for $\ell=2, \ldots, L$, with $\Delta x_{L}$ given. The mapping between vectors defined on two consecutive grids $\Omega_{\ell}$ and $\Omega_{\ell-1}$ is performed by two linear operators: the restriction operator $\mathcal{I}_{\ell}^{\ell-1}$ and the prolongation operator $\mathcal{I}_{\ell-1}^{\ell}$. In our numerical implementations, these operators are linear interpolation operators between the two grids.

To better illustrate the FAS scheme, we consider a general nonlinear problem $A(w)=f$, and denote its discretization by

$$
A_{\ell}\left(w_{\ell}\right)=f_{\ell},
$$

where $A_{\ell}(\cdot)$ is a restriction of the nonlinear operator $A$ to the grid $\Omega_{\ell}$. We assume that a smoothing procedure is available and denoted by $w \mapsto v=$ $B(w, f)$. Now, suppose to apply few times the smoothing map $B$ to (34) and obtain an approximation $\widetilde{w}_{\ell}$ to $w_{\ell}$. The correction $e_{\ell}$ that would lead to the exact solution would be a vector $e_{\ell}$ defined by $A_{\ell}\left(\widetilde{w}_{\ell}+e_{\ell}\right)=f_{\ell}$. We clearly have that $w_{\ell}=\widetilde{w}_{\ell}+e_{\ell}$. If we define $r_{\ell}:=f_{\ell}-A_{\ell}\left(\widetilde{w}_{\ell}\right)$, the correction equation can be written in the form

$$
A_{\ell}\left(\widetilde{w}_{\ell}+e_{\ell}\right)-A_{\ell}\left(\widetilde{w}_{\ell}\right)=r_{\ell},
$$

which for a given $\widetilde{w}_{\ell}$ is solved by the correction $e_{\ell}$. However, the solution of (35) requires the same computational effort needed to solve the original problem (34). On the other hand, the smoothing property of $B$ allows us to well represent $e_{\ell}$ on the coarse grid $\Omega_{\ell-1}$. Now, we define

$$
\widehat{w}_{\ell-1}:=\mathcal{I}_{\ell}^{\ell-1} \widetilde{w}_{\ell}+e_{\ell-1}
$$

and seek a correction equation that can be used to obtain $e_{\ell-1}$, the approximation to $e_{\ell}$ on $\Omega_{\ell-1}$. To do so, we formulate (35) on the coarse level $\Omega_{\ell-1}$ by replacing $A_{\ell}(\cdot)$ by $A_{\ell-1}(\cdot), \widetilde{w}_{\ell}$ by $\mathcal{I}_{\ell}^{\ell-1} \widetilde{w}_{\ell}$, and $r_{\ell}$ by $\mathcal{I}_{\ell}^{\ell-1} r_{\ell}=\mathcal{I}_{\ell}^{\ell-1}\left(f_{\ell}-A_{\ell}\left(\widetilde{w}_{\ell}\right)\right)$. We get the FAS equation

$$
A_{\ell-1}\left(\widehat{w}_{\ell-1}\right)=\mathcal{I}_{\ell}^{\ell-1}\left(f_{\ell}-A_{\ell}\left(\widetilde{w}_{\ell}\right)\right)+A_{\ell-1}\left(\mathcal{I}_{\ell}^{\ell-1} \widetilde{w}_{\ell}\right),
$$

which we write in the form

$$
A_{\ell-1}\left(\widehat{w}_{\ell-1}\right)=\mathcal{I}_{\ell}^{\ell-1} f_{\ell}+\tau_{\ell}^{\ell-1}
$$

where $\tau_{\ell}^{\ell-1}=A_{\ell-1}\left(\mathcal{I}_{\ell}^{\ell-1} \widetilde{w}_{\ell}\right)-\mathcal{I}_{\ell}^{\ell-1} A_{\ell}\left(\widetilde{w}_{\ell}\right)$. 
Once the solution $\widehat{w}_{\ell-1}$ to $(37)$ is obtained, mimicking the exact correction $w_{\ell}=\widetilde{w}_{\ell}+e_{\ell}$, we use (36) and define the correction of $\widetilde{w}_{\ell}$ by

$$
\begin{aligned}
w_{\ell} & =\widetilde{w}_{\ell}+\mathcal{I}_{\ell-1}^{\ell} e_{\ell-1} \\
& =\widetilde{w}_{\ell}+\mathcal{I}_{\ell-1}^{\ell}\left(\widehat{w}_{\ell-1}-\mathcal{I}_{\ell}^{\ell-1} \widetilde{w}_{\ell}\right) .
\end{aligned}
$$

Next, we show how to combine the FAS scheme with Algorithms 1, 2 or 4 (used as smoothers). To do so, we recall the operator $S$ defined in (8) and rewrite it as $S(V)=\mathrm{Op}(V)+F$, where

$$
\mathrm{Op}(V):=\min _{u \in U} e^{-\Delta t} \mathcal{I}[V](x+\Delta t f(x, u)) \text { and } F:=1-e^{-\Delta t} .
$$

Therefore, the fixed-point problem (9) is equivalent to

$$
V-\mathrm{Op}(V)=F
$$

and at each level $\ell$ the corresponding equation is written as $V_{\ell}-\mathrm{Op}_{\ell}\left(V_{\ell}\right)=F_{\ell}$, where $\mathrm{Op}_{\ell}$ and $F_{\ell}$ denote the restriction of $\mathrm{Op}$ and $F$ to the grid $\Omega_{\ell}$. We summarize the FAS method for solving (40) in Algorithm 6. The multilevel

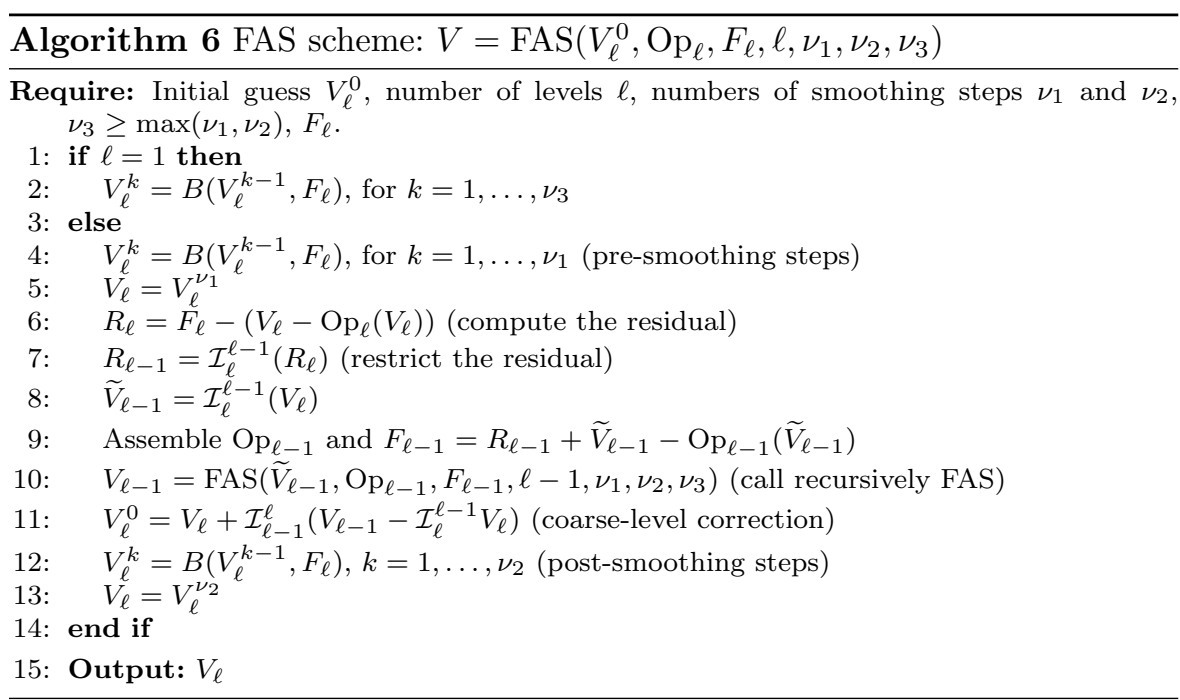

Algorithm 6 represents a so-called V-cycle. We need to discuss further issues related to Algorithm 6.

1. The smoothing procedure $B$ is considered one of Algorithms 1, 2 and 4 .

2. When dealing with nonlinear problems it is important to initialize iterative procedure using a good initial approximation $V^{0}$. An efficient way to get this approximation is to solve the discretized problem (40) on the coarse level and interpolate this solution to the next finer level. This process begins at level 1 and terminate at level $L$. This leads to the so-called Cascadic procedure; see, e.g., [7]. 
3. In step 4 and step 12 we perform only $\nu_{1}$ and $\nu_{2}$ smoothing iterations. Hence, the output $V_{\ell}$ is an approximation of the exact solution. For this reason, one has to repeat several $\mathrm{V}$-cycle. The procedure is stopped when the norm of the residual is smaller than a fixed tolerance.

We summarize our new multilevel strategy in Algorithm in 7.

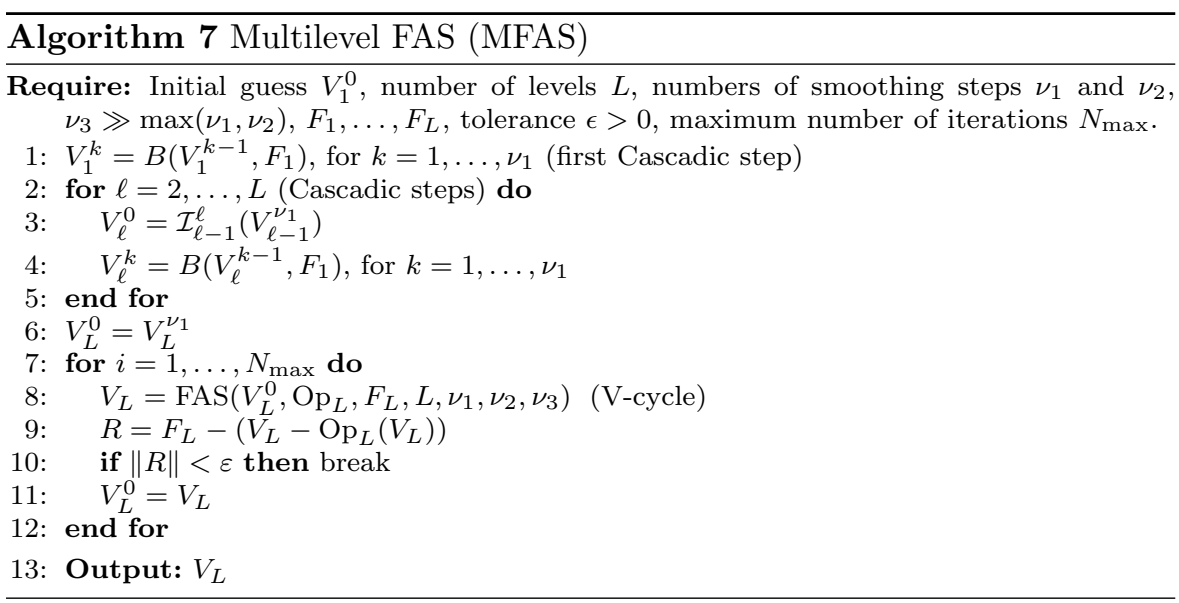

\section{Numerical experiments}

In this section, we present extensive numerical results on the performance of the proposed MFAS method and we compare the results with the solutions given by the classical value iteration algorithm, policy iteration and Anderson acceleration. In all the tests we choose a computational domain $\Omega$ discretized in equidistant nodes with step size $\Delta x$. As discussed in Section 3, the value function will converge to 1 provided that the computational domain is large enough. For this reason, we add some extra-points to our domain with nonuniform step-size $\widetilde{\Delta x}>\Delta x$ and we define this enlarged domain as $\bar{\Omega}$. It is not restrictive to set as boundary conditions on our enlarged domain the constant value 1 , i.e. $\left.v\right|_{\bar{\Omega}} \equiv 1$. Moreover, we always discretize the set of admissible control $U$ with equidistant points. Concerning the parameters involved in the MFAS, we always set $\nu_{1}=\nu_{2}=\nu_{3}=20$. Finally, in all the tests we set as tolerance tol $=10^{-5}$.

Test 1. Van der Pol dynamics

We consider the two-dimensional non-linear dynamics of the Van der Pol oscillator:

$$
f(x, y, u)=\left[\begin{array}{c}
y \\
\left(1-x^{2}\right) y-x+u
\end{array}\right]
$$


The computational domain $\Omega$ is $[-5,5]^{2}$ and discretized with a uniform grid of step size $\Delta t=0.1$. The control set $U$ is $[-1,1]$ and discretized by 32 uniform points. The target set is $\mathcal{T}=(0,0)$. These parameters are summarized in Table 1 .

\begin{tabular}{c|c|c|c|c}
$U$ & controls & $\Omega$ & $\Delta t$ & $\mathcal{T}$ \\
\hline$[-1,1]$ & 32 & {$[-5,5]^{2}$} & 0.01 & $(0,0)$ \\
\hline
\end{tabular}

Table 1 Test 1 (Van der Pol oscillator): parameters choice.

The performance of the methods in terms of CPU time and number of iterations is shown in Table 2. Concerning the implementation of MFAS, we point out that the results showed in the first line of Table 2 refers to the case where the multilevel is performed between two grids, a coarse one with $95^{2}$ and a fine one with $175^{2}$ nodes. In the same way, the results showed in the second and the third lines are obtained using in the MFAS three and four levels, respectively. As expected, we observe that for all the methods (VI, PI and AA) the number of iterations and the CPU time increase as the number of nodes in the grid does. We observe that PI has a faster convergence (in terms of iterations and CPU time) than the other methods, while VI is the slowest. We remark that with coarse grids AA has faster convergence than VI, the speed-up factor is almost 2 (first and second line of Table 2). On the other hand, with the finest grid AA is the slowest. This is due to the fact that the size of the matrices involved in the QR factorization is huge. Hence, despite AA requires less iterations to converge to the solution than VI, each iteration is highly costly. As expected, PI is the fastest method in all the grids. If we compare the results obtained by MFAS we observe a speed up on both the coarse and the fine grids. However, the speed-up factor is bigger on fine grids. When we use PI as a smoother in the MFAS method we have a speed up of factor 2 on the intermediate grid (Table 2 second line) and of factor 3.5 on the finest grid (Table 2 third line). We can observe a similar speed up when we use as smoother VI and AA. In Figure 3 (left) we plot the $L^{\infty}$-norm of the residual for all the methods. It turns out that applying the PI algorithm the decay of the residual is very fast, if compared to the one obtained applying VI or AA. Moreover, we remark that in the MFAS few iterations are needed until the residual becomes smaller than the fixed tolerance $\left(\right.$ tol $\left.=10^{-5}\right)$. We remark that in all the algorithms the residual at iteration $k=1$ (Figure 3 right) is computed after the initialization with the Cascadic procedure, while the residual at iteration $k=2, \ldots$ is computed after each $V$-cycle. 


\begin{tabular}{c|cccccc}
\hline nodes & VI & MFAS+VI & PI & MFAS+PI & AA & MFAS+AA \\
\hline $175^{2}$ & $37.1[591]$ & $31.5[11]$ & $9.8[17]$ & $7.3[2]$ & $16.6[227]$ & $17.1[6]$ \\
\hline $335^{2}$ & $129.5[630]$ & $79.8[7]$ & $59.2[29]$ & $29.7[2]$ & $94.3[354]$ & $43.6[4]$ \\
\hline $655^{2}$ & $526.7[661]$ & $233.2[6]$ & $483.1[67]$ & $137.9[2]$ & $640.1[520]$ & $159.5[4]$ \\
\hline
\end{tabular}

Table 2 Test 1 (Van der Pol oscillator): CPU time and iterations for different algorithms.
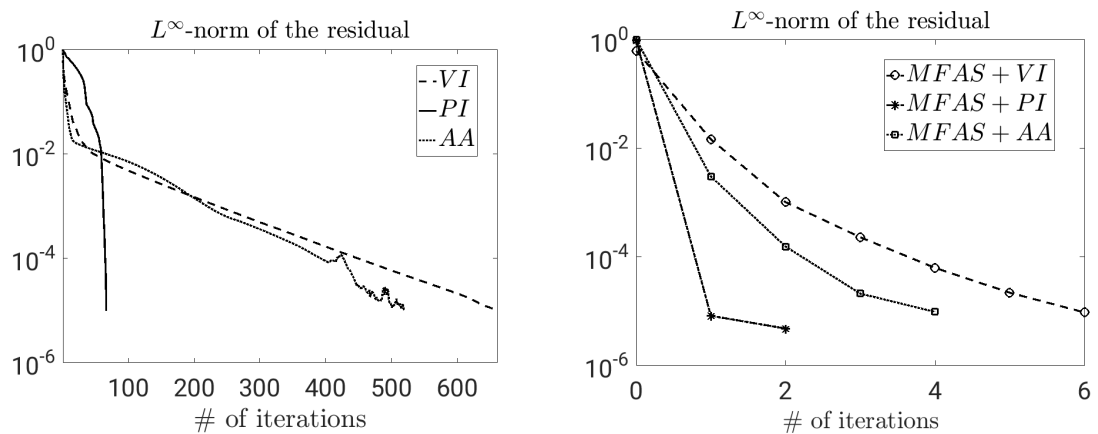

Fig. $3 L^{\infty}$-norm of the residual for the Van der Pol oscillator applying VI, PI, AA (left) and MFAS+VI, MFAS+PI, MFAS+AA (right).

Test 2 and 3. 2D-Eikonal equation

For both tests we consider the dynamics described by the two dimensional eikonal equation:

$$
f(x, y, u)=\left[\begin{array}{l}
\cos (u) \\
\sin (u)
\end{array}\right]
$$

\begin{tabular}{c|c|c|c|c|c} 
& $U$ & controls & $\Omega$ & $\Delta \mathrm{t}$ & $\mathcal{T}$ \\
\hline Test 2 & {$[-\pi, \pi]$} & 64 & {$[-5,5]^{2}$} & 0.01 & $(0,0)$ \\
Test 3 & {$[-\pi, \pi]$} & 72 & {$[-5,5]^{2}$} & 0.01 & $\left\{x \in \mathbb{R}^{2}:\|x\|_{2} \leq 1\right\}$ \\
\hline
\end{tabular}

Table 3 Tests 2 and 3 (2D-Eikonal equation): parameters choice

The solution of the eikonal equation can be interpreted as the distance function to the target. In Test 2 we consider as a target a single point, the origin $\mathcal{T}=(0,0)$. The choice of the domain $\Omega$ as well as the target $\mathcal{T}$, the step size $\Delta t$ and the control set $U$ are summarized in Table 3 . When we use VI as smoother for the MFAS scheme we observe a speed-up of factor 2 in the fine grid (Table 4, third line). On the other hand, when we use PI or AA as smoothers the speed-up factor is significantly bigger, 5 and 6 respectively. In Test 3 we choose a more complex target, a unit sphere centered in $(0,0)$ and we increase the number of controls. As expected the CPU times increases for all the algorithms (Table 5). However, the speed-up factor using MFAS is the same as Test 2 as shown in Table 5 . In fact, the speed of a multilevel method does not deteriorate when the discretization is refined or when the dynamics 


\begin{tabular}{c|cccccc}
\hline nodes & VI & MFAS+VI & PI & MFAS+PI & AA & MFAS+AA \\
\hline $175^{2}$ & $50[662]$ & $45.2[12]$ & $14.9[87]$ & $10.7[2]$ & $14.4[174]$ & $11.1[3]$ \\
\hline $335^{2}$ & $140.4[701]$ & $88.5[8]$ & $64.3[167]$ & $23.3[2]$ & $71[284]$ & $26.5[3]$ \\
\hline $655^{2}$ & $503.6[732]$ & $215.4[6]$ & $359.8[327]$ & $68[2]$ & $505.9[470]$ & $81.4[3]$ \\
\hline
\end{tabular}

Table 4 Test 2 (2D-Eikonal equation): CPU time and iterations for different algorithms.

\begin{tabular}{c|cccccc} 
nodes & VI & MFAS+VI & PI & MFAS+PI & AA & MFAS+AA \\
\hline $175^{2}$ & $56.4[657]$ & $50[12]$ & $14.7[75]$ & $10.1[2]$ & $15.5[161]$ & $11.4[3]$ \\
\hline $335^{2}$ & $157.57[678]$ & $101.7[8]$ & $64.3[144]$ & $25[2]$ & $71.29[259]$ & $30.4[3]$ \\
\hline $655^{2}$ & $544.74[689]$ & $234.4[6]$ & $365.3[281]$ & $51[2]$ & $484.5[423]$ & $106[3]$ \\
\hline
\end{tabular}

Table 5 Test 3 (2D-Eikonal equation): CPU time and iterations for different algorithms.

is more complex. In Figure 4 we show the contour lines of the computed value functions corresponding to Test 2 (left) and Test 3 (right).
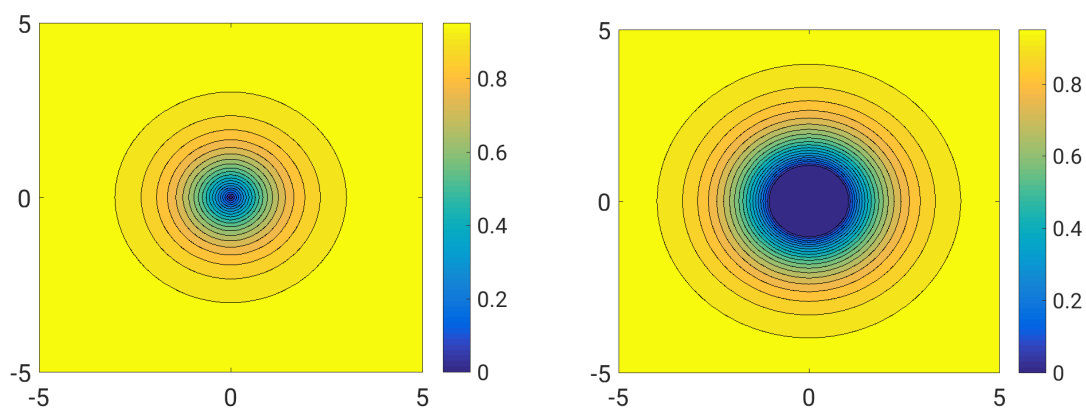

Fig. 4 Contour lines of the value function for 2D-eikonal equation: Test 2 (left), Test 3 (right).

Test 4. Zermelo Navigation Problem

We consider the Zermelo navigation problem which deals with a boat navigating on a body of water, originating from a point $\mathrm{A}$ to a destination point $\mathrm{B}$. The boat is capable of a certain maximum speed, and the goal is to derive the best possible control to reach $\mathrm{B}$ in the least possible time. The dynamics is given by

$$
f(x, y, u)=\left[\begin{array}{c}
1+V_{b} \cos (u) \\
V_{b} \sin (u)
\end{array}\right]
$$

The choice of the domain $\Omega$ as well as the target $\mathcal{T}$, the step size $\Delta t$ and the control set $U$ are summarized in Table 6 .

In (43) we can control the angle of the boat $u$, while the velocity is fixed, $V_{b}=$ 1.8. The results of the simulations are summarized in Table 7. As observed in the previous experiments, the speed-up factor is almost 7 using PI as smoother 


\begin{tabular}{c|c|c|c|c}
$U$ & controls & $\Omega$ & $\Delta \mathrm{t}$ & $\mathcal{T}$ \\
\hline$[-\pi, \pi]$ & 72 & {$[-9,9]^{2}$} & 0.01 & $\left\{x \in \mathbb{R}^{2}:\|x\|_{2} \leq 0.2\right\}$ \\
\hline
\end{tabular}

Table 6 Test 4 (Zermelo navigation problem): parameters choice
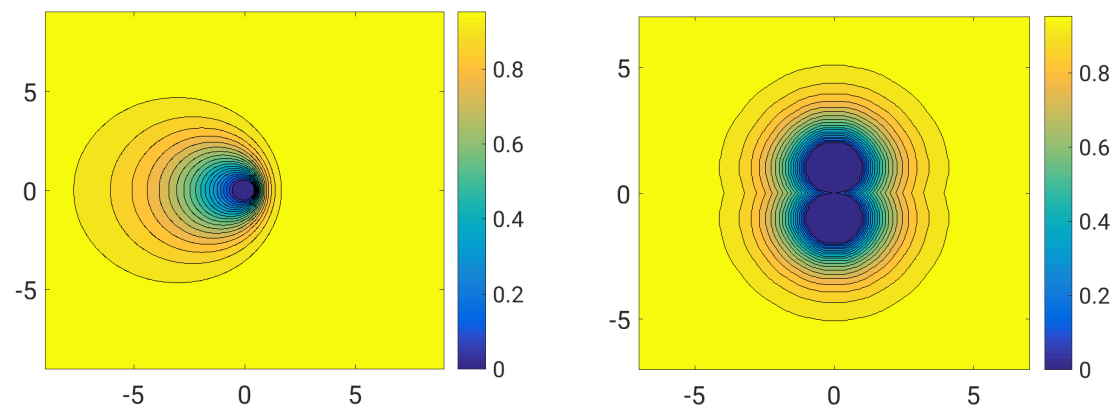

Fig. 5 Contour lines of the value function for the Zermelo navigation problem (left), contour plot of the value function at $z=0$ for the $3 D$-Eikonal equation with target $\widetilde{\mathcal{T}}$ (right).

in the MFAS method on the finest grid. In Figure 5 we show the contour lines

\begin{tabular}{c|cccccc} 
nodes & VI & MFAS+VI & PI & MFAS+PI & AA & MFAS+AA \\
\hline $175^{2}$ & $102.8[657]$ & $111.3[12]$ & $35.8[84]$ & $23.6[2]$ & $39.7[187]$ & $25.8[3]$ \\
\hline $335^{2}$ & $433.9[688]$ & $176.4[8]$ & $209[160]$ & $48.4[2]$ & $255.7[307]$ & $58.8[3]$ \\
\hline $655^{2}$ & $1146.2[708]$ & $462.5[6]$ & $937.6[312]$ & $133.5[2]$ & $817.5[643]$ & $290.6[3]$ \\
\hline
\end{tabular}

Table 7 Test 4 (Zermelo navigation problem): CPU time and iterations for different algorithms.

of the value function (left).

Test 5 and 6. 3D-Eikonal equation

We develop a three-dimensional extension of the dynamics (42). We consider the dynamics given by the 3D-eikonal equation:

$$
f(x, y, z, u)=\left[\begin{array}{c}
\sin \left(u_{1}\right) \cos \left(u_{2}\right) \\
\sin \left(u_{1}\right) \sin \left(u_{2}\right) \\
\cos \left(u_{1}\right)
\end{array}\right] .
$$

The choice of the domain $\Omega$ as well as the target $\mathcal{T}$, the step size $\Delta t$ and the control set $U$ are summarized in Table 8 . In Test 5 we consider as a target the origin $(0,0,0)$, while in Test 6 our target is the union of two unit spheres centered in $(-1,0,0)$ and $(1,0,0)$, i.e. $\widetilde{\mathcal{T}}:=\left\{x \in \mathbb{R}^{3}:\|x-(-1,0,0)\|_{2} \leq\right.$ $\left.1 \wedge\|x-(1,0,0)\|_{2} \leq 1\right\}$. When the dimension of the state variable increases and we consider a fine grid the computational cost explode and the CPU time 


\begin{tabular}{c|c|c|c|c|c} 
& $U$ & controls & $\Omega$ & $\Delta \mathrm{t}$ & $\mathcal{T}$ \\
\hline Test 5 & {$[-\pi, \pi] \times[0, \pi]$} & $16 \times 8$ & {$[-7,7]^{3}$} & 0.01 & $(0,0,0)$ \\
\hline Test 6 & {$[-\pi, \pi] \times[0, \pi]$} & $16 \times 8$ & {$[-7,7]^{3}$} & 0.01 & $\widetilde{\mathcal{T}}$ \\
\hline
\end{tabular}

Table 8 Test 5 and 6 (3D Eikonal equation): parameters choice

of all the algorithms drastically increases. Because of the dimension and the huge number of nodes, the AA method fails for out-of-memory error. Hence, in this case the use of MFAS turns out to be crucial. We can efficiently use AA as a smoother for the MFAS method as shown in Tables 9 and 10. The speed-up

\begin{tabular}{r|cccccc} 
nodes & VI & MFAS+VI & PI & MFAS+PI & AA & MFAS+AA \\
\hline $95^{3}$ & $2157.8[617]$ & $1686.3[11]$ & $365.5[46]$ & $104.1[2]$ & $351.6[90]$ & $264.41[2]$ \\
\hline $175^{3}$ & $15555[652]$ & $7392[7]$ & $3851.9[85]$ & $483.2[2]$ & - & $1579.2[2]$ \\
\hline
\end{tabular}

Table 9 Test 5 (3D eikonal equation): CPU time and iterations for different algorithms.

\begin{tabular}{r|cccccc} 
nodes & VI & MFAS+VI & PI & MFAS+PI & AA & MFAS+AA \\
\hline $95^{3}$ & $2194.1[618]$ & $1698.8[11]$ & $345.3[42]$ & $128[2]$ & $394.5[94]$ & $246.4[2]$ \\
\hline $175^{3}$ & $15803[653]$ & $7995.5[8]$ & $3665.6[76]$ & $776.9[2]$ & - & $2452.8[3]$ \\
\hline
\end{tabular}

Table 10 Test 6 (3D eikonal equation): CPU time and iterations for different algorithms.

factor using PI as smoother for MFAS is 6, the same that we observed in the 2D-experiments. This indicates that the speed of a multilevel method does not deteriorate when the discretization is refined, whereas classical iterative methods slow down for decreasing grid size.

The contour plot of the value function at $z=0$ obtained in Test 6 are shown in Figure 5 (right).

Test 7. Heat equation

We consider the dynamics given by the linear heat equation

$$
y_{t}=\nu \Delta y \quad \text { in }[0, T] \times Q, \quad \frac{\partial y}{\partial n}=u(t) \quad \text { in }[0, T] \times \partial Q, \quad y(0)=y_{0} .
$$

where $Q=[0,1]^{2}$ and $\nu=1 / 10$. We use a finite element space of piecewise linear basis function for the spatial approximation of a weak solution to (45). This leads to a finite-dimensional linear system. Hence, we perform a Galerkin projection with the basis function computed via Proper Orthogonal Decomposition (POD, see [15] and the reference therein for further details) to obtain a low dimensional system. In this test we choose the first 4 reduced states which 
provide a good description of the full system. This leads to the following reduced system:

$$
f(x, y, z, w, u)=\left[\begin{array}{c}
-0.0008 x-0.0593 y-0.0212 z-0.0196 w-3.9535 u \\
-0.0593 x-4.2695 y-2.8948 z-2.749 w+3.5394 u \\
-0.0212 x-2.8948 y-35.9197 z-44.5585 w+6.4529 u \\
-0.0196 x-2.7490 y-44.5585 z-125.8934 w+7.7194 u
\end{array}\right] .
$$

The choice of the domain $\Omega$ as well as the target $\mathcal{T}$, the step size $\Delta t$ and the control set $U$ are summarized in Table 11. Moreover, we set $\nu_{1}=\nu_{2}=\nu_{3}=10$ in the MFAS algorithm. As already seen in Test 5 and 6 when the dimension of

\begin{tabular}{c|c|c|c|c}
$U$ & controls & $\Omega$ & $\Delta t$ & $\mathcal{T}$ \\
\hline$[-2,2]$ & 17 & {$[-2,2]^{2}$} & 0.01 & $(0,0,0,0)$ \\
\hline
\end{tabular}

Table 11 Test 7 (Heat equation): parameters choice.

the state variable increase, the computational cost explode. If we want a good approximation of the value function on the computational domain, one should consider a space step $\Delta x \leq 5 \cdot 10^{-2}$. Choosing $\Delta x=5 \cdot 10^{-2}$, the VI algorithm requires almost 10 hours to converge. However, with our multilevel approach the speed-up factor is almost two (Table 12 second and fourth column). We remark that because of the huge number of nodes the AA algorithm fails for out-of-memory error.

\begin{tabular}{r|cccc} 
nodes & VI & MFAS+VI & PI & MFAS+PI \\
\hline $81^{4}$ & $37^{\prime} 390[174]$ & $22^{\prime} 960[6]$ & $14^{\prime} 461[24]$ & $8627.5[2]$ \\
\hline
\end{tabular}

Table 12 Test 7 (heat equation): CPU time and iterations for different algorithms.

Finally in Figure 6 we show the contour lines of the value function at $z=w=0$ (left) and at $y=w=0$ (right).

\section{Conclusions}

In this work, we developed two new iterative acceleration procedures for the efficient numerical solution of minimum-time control problems in the framework of the Hamilton-Jacobi equation. The first strategy is obtained by accelerating the well-known value iteration using the so-called Anderson acceleration method. Hence, we extended the existing convergence results to the nondifferentiable case of the Hamilton-Jacobi equation. We have shown then that this new scheme and other classical iterative schemes are efficient smoothers. This allowed us to combine them with a FAS multilevel strategy to obtain new very fast numerical methods. We illustrated the efficiency of our new numerical scheme with several numerical experiments. 

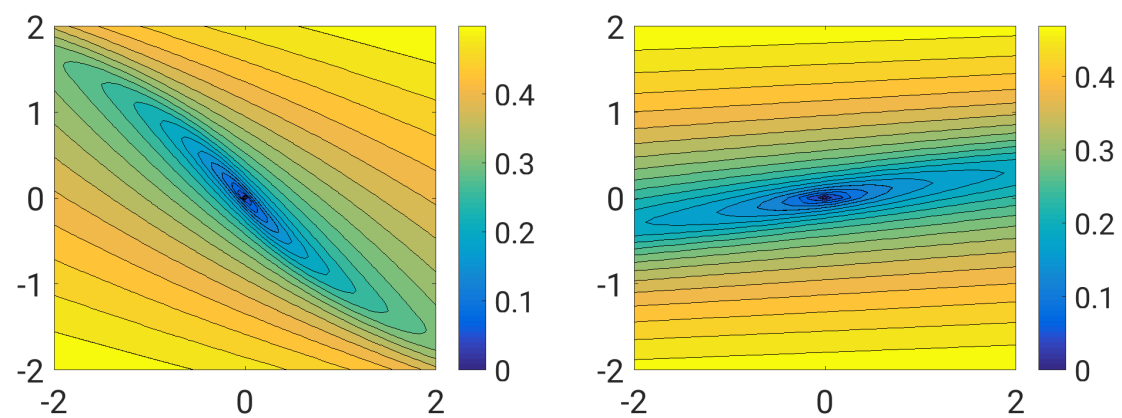

Fig. 6 Contour lines of the value function for the heat equation at $z=w=0$ (left) and at $y=w=0$ (right).

\section{References}

1. Alla, A., Falcone, M., Kalise, D.: An efficient policy iteration algorithm for dynamic programming equations. SIAM Journal on Scientific Computing 37(1), A181-A200 (2015)

2. Anderson, D.: Iterative procedures for nonlinear integral equations. J. Assoc. Comput. Mach. 12, 547 (1965)

3. Bardi, M., Dolcetta, I.C.: Optimal control and viscosity solutions of Hamilton-JacobiBellman equations. Birkhäuser, Basel (1997)

4. Bardi, M., Falcone, M.: An approximation scheme for the minimum time function. SIAM Journal on Control and Optimization 28(4), 950-965 (1990)

5. Bellman, R.: Dynamic programming. Princeton University Press, Princeton, NJ (1957)

6. Borzì, A.: Introduction to Multigrid Methods. Lecture Notes. (2003)

7. Borzì, A., Ciaramella, G., Sprengel, M.: Formulation and Numerical Solution of Quantum Control Problems. Society for Industrial and Applied Mathematics, Philadelphia, PA (2017). DOI 10.1137/1.9781611974843

8. Cacace, S., Cristiani, E., Falcone, M., Picarelli, A.: A patchy dynamic programming scheme for a class of hamilton-jacobi-bellman equations. SIAM Journal on Scientific Computing 34(5), A2625-A2649 (2012)

9. Camilli, F., Falcone, M., Lanucara, P., Seghini, A.: A domain decomposition method for bellman equations. Contemporary Mathematics 180, 477-477 (1994)

10. Falcone, M.: Numerical solution of dynamic programming equations. Optimal Control and Viscosity Solutions of Hamilton-Jacobi-Bellman equations. Birkhäuser (1997)

11. Falcone, M., Ferretti, R.: Semi-Lagrangian approximation schemes for linear and Hamilton-Jacobi equations, vol. 133. SIAM (2014)

12. Festa, A.: Reconstruction of independent sub-domains for a class of hamilton-jacobi equations and application to parallel computing. ESAIM: Mathematical Modelling and Numerical Analysis 50(4), 1223-1240 (2016)

13. Fleming, W., Rishel, R.: Deterministic and sthochastic optimal control. Springer Verlag, New York, (1975)

14. Gander, W., Gander, M.J., Kwok, F.: Scientific computing-An introduction using Maple and MATLAB, vol. 11. Springer Science \&amp; Business (2014)

15. Gubisch, M., Volkwein, S.: Proper orthogonal decomposition for linear-quadratic optimal control. Model Reduction and Approximation: Theory and Algorithms. SIAM, Philadelphia, PA (2017)

16. Hackbusch, W.: Multi-Grid Methods and Applications. Springer (2003)

17. Henson, V.E.: Multigrid methods for nonlinear problems: an overview. Tech. rep., Lawrence Livermore National Lab., CA (US) (2002)

18. Howard, R.A.: Dynamic programming. Management Science 12(5), 317-348 (1966)

19. Jurdjevic, V.: Geometric Control Theory. Cambridge Studies in Advanced Mathematics. Cambridge University Press (1996) 
20. Lee, E.B., Markus, L.: Foundations of Optimal Control Theory. Wiley, New York (1967)

21. Pollatschek, M., Avi-Itzhak, B.: Algorithms for stochastic games with geometrical interpretation. Management Science 15(7), 399-415 (1969)

22. Puterman, M.L., Brumelle, S.L.: On the convergence of policy iteration in stationary dynamic programming. Mathematics of Operations Research 4(1), 60-69 (1979)

23. Saad, Y.: Numerical Methods for Large Eigenvalue Problems. Manchester University Press, Manchester, UK (1992)

24. Santos, M.S., Rust, J.: Convergence properties of policy iteration. SIAM Journal on Control and Optimization 42(6), 2094-2115 (2004)

25. Sussmann, H.J., Willems, J.C.: 300 years of optimal control: from the brachystochrone to the maximum principle. IEEE Control Systems Magazine 17, 32-44 (1997)

26. Toth, A., Kelley, C.: Convergence analysis for Anderson acceleration. SIAM Journal on Numerical Analysis 53(2), 805-819 (2015)

27. Ulbrich, M.: Semismooth Newton Methods for Variational Inequalities and Constrained Optimization Problems in Function Spaces, vol. 11. SIAM (2011)

28. Walker, H.F.: Anderson acceleration: algorithms and implementations. WPI Math. Sciences Dept. Report MS-6-15-50 (2011)

29. Walker, H.F., Ni, P.: Anderson acceleration for fixed-point iterations. SIAM Journal on Numerical Analysis 49(4), 1715-1735 (2011) 